\title{
Alterations in white matter network topology contribute to freezing of gait in Parkinson's disease
}

\author{
Julie M. Hall ${ }^{1,2} \cdot$ James M. Shine ${ }^{2} \cdot$ Kaylena A. Ehgoetz Martens ${ }^{2} \cdot$ Moran Gilat $^{2} \cdot$ Kathryn M. Broadhouse ${ }^{2}$. \\ Jennifer Y. Y. Szeto ${ }^{2}$. Courtney C. Walton ${ }^{2}$. Ahmed A. Moustafa ${ }^{1,3} \cdot$ Simon J. G. Lewis ${ }^{2}$
}

Received: 2 February 2018 / Revised: 16 March 2018 / Accepted: 21 March 2018 / Published online: 3 April 2018

c) Springer-Verlag GmbH Germany, part of Springer Nature 2018

\begin{abstract}
Freezing of gait (FOG) is a common symptom in advanced Parkinson's disease (PD). Despite current advances, the neural mechanisms underpinning this disturbance remain poorly understood. To this end, we investigated the structural organisation of the white matter connectome in PD freezers and PD non-freezers. We hypothesized that freezers would show an altered network architecture, which could hinder the effective information processing that characterizes the disorder. Twentysix freezers and twenty-four well-matched non-freezers were included in this study. Using diffusion tensor imaging, we investigated the modularity and integration of the regional connectome by calculating the module degree $z$ score and the participation coefficient, respectively. Compared to non-freezers, freezers demonstrated lower participation coefficients in the right caudate, thalamus, and hippocampus, as well as within superior frontal and parietal cortical regions. Importantly, several of these nodes were found within the brain's 'rich club'. Furthermore, group differences in module degree $z$ scores within cortical frontal and sensory processing areas were found. Together, our results suggest that changes in the structural network topology contribute to the manifestation of FOG in PD, specifically due to a lack of structural integration between key information processing hubs of the brain.
\end{abstract}

Keywords Parkinson's disease $\cdot$ Freezing of gait $\cdot$ Connectome $\cdot$ Diffusion imaging

\section{Introduction}

Freezing of gait (FOG) is a sudden and involuntary gait cessation that is common in idiopathic Parkinson's disease (PD) [1] and has a prominent impact on quality of life [2]. Freezing behaviour can be provoked by a range of triggers, including turning, dual tasking, and emotional stress [3]. Treatment options are limited, and although dopaminergic

Electronic supplementary material The online version of this article (https://doi.org/10.1007/s00415-018-8846-3) contains supplementary material, which is available to authorized users.

Simon J. G. Lewis

profsimonlewis@gmail.com

1 School of Social Sciences and Psychology, Western Sydney University, Milperra, NSW 2214, Australia

2 Brain and Mind Centre, University of Sydney, 100 Mallett Street, Camperdown, NSW 2050, Australia

3 MARCS Institute, Western Sydney University, Milperra, NSW 2214, Australia medication can partially ameliorate freezing, patients are often forced to rely on behavioural strategies that temporarily enhance their gait pattern using goal-directed strategies [1]. Furthermore, patients with freezing often experience executive dysfunction, mood disorders, and perceptual problems [4-6]. These observations have led to the suggestion that FOG is not simply the result of dysfunctional locomotor output, but rather results from a diffuse, network-level dysfunction that ultimately manifests as a loss of top-down control over the brainstem structures that control gait [7].

A range of studies has been conducted to determine whether individuals with FOG demonstrate white matter abnormalities. For instance, widespread alterations in structural connections between cortical, subcortical, and brainstem regions have been reported in studies using diffusion tensor imaging (DTI), a technique that infers the integrity of inter-regional white matter. Compared to non-freezers, freezers have shown abnormalities in the corticopontine, pontine-cerebellar [8-11], and major cortico-cortical and frontostriatal tracts $[10,12]$. Conversely, differences were not observed in a study investigating the frontostriatal 
hyper-direct pathway [13] or in a whole-brain analysis between freezers and non-freezers [14]. These results suggest that structural lesions in freezers might be subtle and dispersed, and hence raise the question of whether FOG actually stems from a focal anatomical lesion at all [7]. Indeed, a key caveat of the majority of studies to date is that they have used diffusion imaging in an attempt to identify lesions within specific regions or tracts. As such, they offer insights that are relatively limited to the field of interest and, therefore, miss more complex, distributed patterns that might exist in the data.

Given the multi-dimensional nature of FOG, the primary objective of this study was to investigate the structural brain network architecture in patients with FOG and this debilitating phenomenon. Specifically, we investigated whether white matter changes that contribute to freezing might emerge as alterations in structural network topology. The brain displays a modular structure, in which discrete communities ('modules') with densely interconnected nodes are only sparsely connected with nodes of other communities $[15,16]$. These specialist modules allow for processing without unnecessary cross-talk [17]. Conversely, the integration of communities is crucial for incorporating complex information streams of different modalities, such as in cognitively challenging tasks (Fig. 1b) $[15,18]$.

Graph theory offers a robust mathematical framework for interrogating neural network topology, and can offer a detailed in vivo analysis of the brain at the level of circuits and modules [19]. Importantly, whole-brain graph theoretical embraces the inherent complexity of interacting subsystems, rather than isolating a particular region within a circuit. Here, we used a combination of DTI and graph theoretical analyses to investigate the global and regional topology of the structural connectome in individuals with PD and FOG. Given the known impairments in multi-domain behaviour in patients with freezing, we predicted that freezers should exhibit impairments in inter-modular connectivity, especially in frontoparietal and subcortical networks [7].

\section{Materials and methods}

Fifty patients with mild-to-moderate idiopathic PD, recruited from the Parkinson's Disease Research Clinic at the Brain and Mind Centre, University of Sydney, were included in this study. Demographic and clinical details including age, gender, disease duration, levodopa equivalent daily dose, and motor severity were obtained and are listed in Table 1. Motor severity was measured by the Movement Disorder Society Unified Parkinson's Disease Rating Scale (MDS-UPDRS) part III [20] and Hoehn and Yahr (H\&Y) scale [21]. Patients with a $H \& Y$ score of $>3$ were excluded from the study. Cognitive screening was performed using the Mini-Mental State Examination [22] and patients with a score of $\leq 25$ on this test were also excluded from the study. Finally, Logical Memory [23], the Trail Making Test Part $\mathrm{B}-\mathrm{A}\left(\mathrm{TMT}_{\mathrm{B}-\mathrm{A}}\right)$ [24], and Digit Span Backward (DSB) [23] were used as measures of memory and executive functioning ability. Patients were identified as 'freezers' if they scored positively on the Freezing of Gait Questionnaire item 3 [25, $26]$ and as 'non-freezers' if they scored 0 on this item.

\section{MRI acquisition}

Neuroimaging was acquired on a 3-Tesla General Electric Discovery MR750 scanner (GE Medical Systems) using an eight-channel-phased array head coil. 3D T1-weighted, anatomical images (voxel size $1 \times 1 \times 1 \mathrm{~mm}$ ) were obtained for parcellation of cortical regions, using the following protocol: Repetition time $7.2 \mathrm{~ms}$, echo time $2.7 \mathrm{~ms}$, inversion time $450 \mathrm{~ms}$, 196 transverse slices, $256 \times 256$ matrix; field of view $256 \times 256 \mathrm{~mm}$, and flip angle $12^{\circ}$. Diffusion-weighted images (DWI) were acquired in 61-gradient directions, using the following echoplanar imaging protocol: repetition time $7025 \mathrm{~ms}$, echo time $80 \mathrm{~ms}, 55$ transverse slices, $2.5 \mathrm{~mm}$ slice thickness, $256 \times 256$ matrix; field of view $240 \times 240 \mathrm{~mm}$. The effective diffusion weighting was $b=1000 \mathrm{~s} / \mathrm{mm}^{2}$. Four volumes with no diffusion weighting $\left(b=0 \mathrm{~s} / \mathrm{mm}^{2}\right)$ were acquired at the beginning of each diffusion sequence and used for robust individual registration between T1-weighted anatomical and the diffusion-weighted images.

\section{Image pre-processing}

Diffusion image pre-processing was performed using the FMRIB Software Library (FSL, http://fsl.fmrib.ox.ac.uk). Raw DWI were corrected for susceptibility, head motion, and eddy current distortions using FSL's tool $e d d y$ and skull stripped using BET. T1-weighted images were segmented into white and grey matter (cortical and subcortical structures) and cerebrospinal fluid using FreeSurfer (version 5.3; http://surfe r.nmr.mgh.harvard.edu). The cortical surface was further parcellated into 219 cortical regions using the Lausanne anatomical atlas [27] distributed as part of the Connectome Mapping Toolkit [28] and validated for diffusion MRI analysis. Using FreeSurfer, 14 subcortical structures were segmented including the left and right thalamus, caudate, putamen, pallidum, amygdala, hippocampus, and nucleus accumbens area by an atlas based segmentation [29]. This atlas was chosen, because this scale has been shown to provide local interpretability whilst containing homogeneous clusters [27]. T1-weighted images were registered in DWI space to the b0 volume. Fibre reconstruction was performed using robust tensor fitting. The main diffusion direction of each voxel was estimated after decomposition of the tensor into eigenvectors and eigenvalues, and fractional anisotropy values, a measure of magnitude 
(a) Morphological

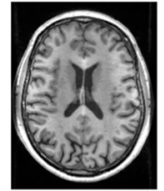

FreeSurfer Segmentation grey, white matter, CSF

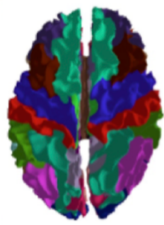

\section{Diffusion}

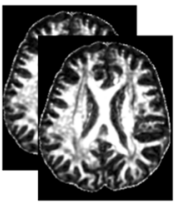

FSL

Eddy

Motion

Correction

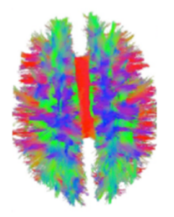

Deterministic tractography

Fractional anisotropy

(b)

\section{Graph Metrics}

Lausanne

219 cortical

and 14

subcortical

ROIs

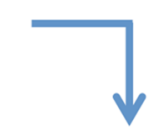

\section{Registration}

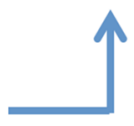

\section{$\underline{\text { Connectome }}$}

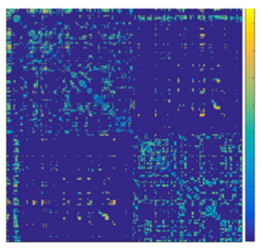

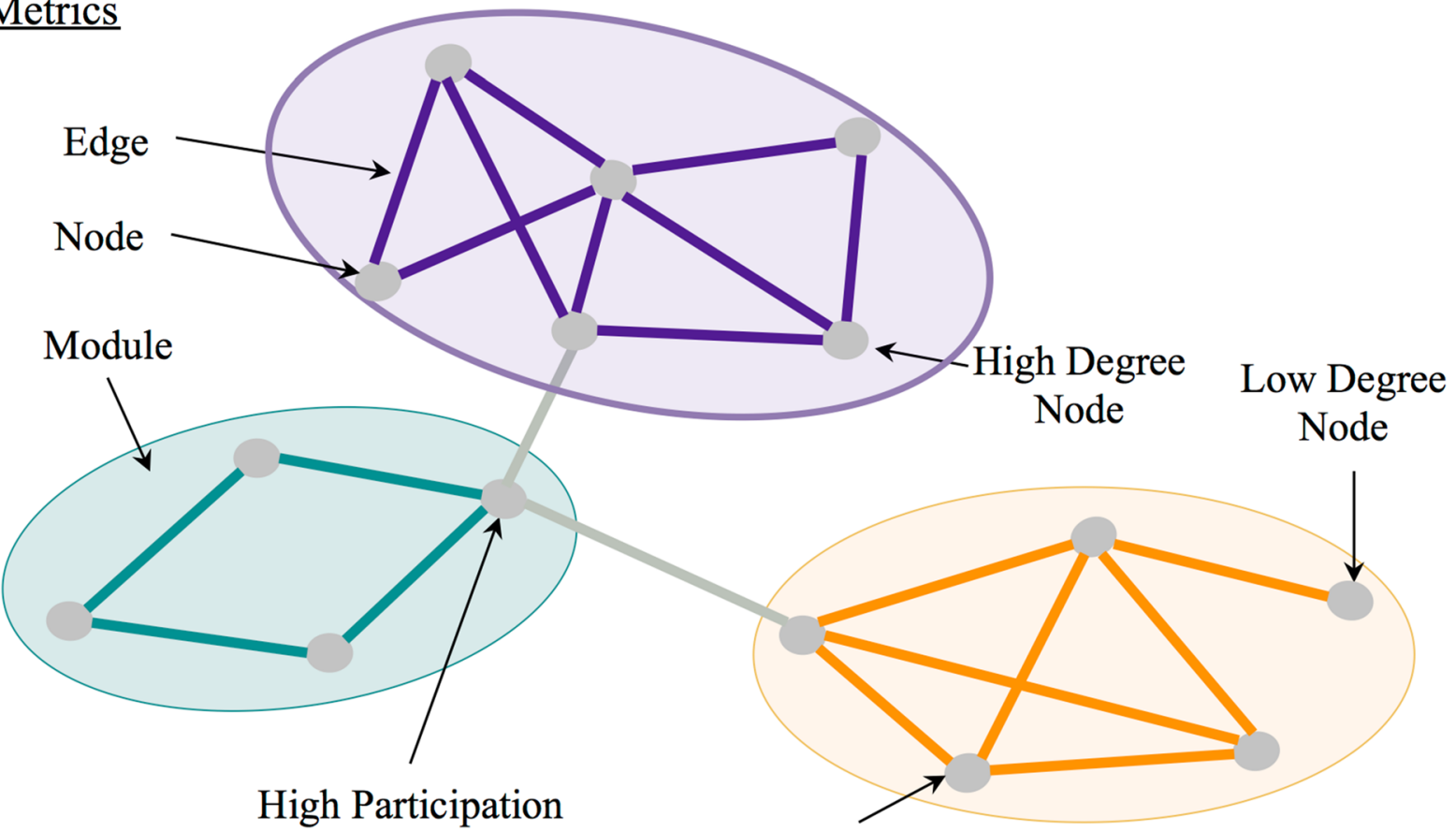

Coefficient Node

High Module

Degree Node

Fig. 1 a Imaging processing flow. b. Representation of a brain network with a modular structure. Different graph metrics are highlighted

of directionally of diffusion in white matter fibres, were computed. White matter pathways were constructed using deterministic fibre tracking approach. The deterministic approach was chosen to control for the number of false positives, which are unfavourable when investigating network topology [30].
Using the fibre assignment by continuous tracking algorithm, streamlines were traced following the main diffusion direction starting from eight seeds evenly distributed in each white matter voxel and stopping when one of the following criteria was met: (1) fractional anisotropy $<0.1$; (2) turn $>45^{\circ}$; (3) 
Table 1 Demographic and clinical data of patients with and without freezing of gait

\begin{tabular}{|c|c|c|c|}
\hline & Freezers & Non-freezers & $p$-value \\
\hline$n$ & 26 & 24 & - \\
\hline Age, years, mean \pm SD & $64.2 \pm 9.4$ & $66.9 \pm 5.3$ & 0.220 \\
\hline Disease duration, years, mean $\pm \mathrm{SD}$ & $5.2 \pm 3.8$ & $5.2 \pm 3.0$ & 0.970 \\
\hline LEDD, mg, mean (range)* & $656.7(0-1548)$ & $526.6(0-1200)$ & 0.431 \\
\hline MDS-UPDRS III, mean \pm SD & $25.5 \pm 13.0$ & $23.3 \pm 13.5$ & 0.558 \\
\hline $\mathrm{H} \& \mathrm{Y}$, mean (range)* & $2.0(1-2.5)$ & $1.8(1-2.5)$ & 0.131 \\
\hline MMSE, mean (range) * & $28.5(26-30)$ & $29.1(26-30)$ & 0.051 \\
\hline Logical Memory I, mean \pm SD & $36.1 \pm 10.0$ & $39.8 \pm 10.3$ & 0.201 \\
\hline Logical Memory II, mean \pm SD & $21.3 \pm 7.8$ & $22.5 \pm 10.4$ & 0.657 \\
\hline $\mathrm{TMT}_{\mathrm{B}-\mathrm{A},}$ sec, mean (range) $*$ & $61.6(17-216)$ & $41.0(-1-92)$ & 0.063 \\
\hline Digit span backward, mean \pm SD & $6.38 \pm 1.8$ & $7.57 \pm 3.3$ & 0.118 \\
\hline
\end{tabular}

LEDD levodopa equivalence daily dose, MDS-UPDRS III motor part of the Movement Disorder Society Unified Parkinsons Disease Rating Scale, $H \& Y$ Hoehn and Yahr motor scale, MMSE mini-mental state examination, $T M T_{B-A}$ trail making test part $\mathrm{B}-\mathrm{A}$

*Mann-Whitney $U$ test the end of the brain mask was reached. Connectome maps were reconstructed using the Connectome Mapper [28]. By combining the parcellation map and the reconstructed fibres, a connectivity matrix was created for each subject, using the average fractional anisotropy of voxels of the reconstructed streamlines. The pipeline is illustrated in Fig. 1a. Finally, to account for spurious streamlines, values of tracts that were present in less than $50 \%$ of all participants were set to zero by applying a threshold on the individual connectivity matrices [31]. To verify the results which were not skewed by the choice of threshold, the analysis was also performed using thresholds of 40 and $60 \%$.

\section{Graph theoretical analyses}

Graph metrics were calculated on the thresholded adjacency matrices using MATLAB scripts from the Brain Connectivity Toolbox [32]. First, we confirmed that the group mean structural connectome demonstrated a 'small-world' architecture, by comparing the ratio of the mean clustering coefficient (the average intensity of triangles around a node) to the shortest path length (a metric of information transmission) of the thresholded connectivity matrix. We next sought to identify significant differences in graph metrics between the two groups. To this end, network modularity was calculated using the Louvain modularity algorithm [32]. This algorithm iteratively assigns nodes to different communities, until the maximum possible modularity statistic $Q$ is reached. In this way, the network is subdivided into non-overlapping groups of highly connected nodes that minimizes between module edges:

$Q=\frac{1}{v} \sum_{i j}\left(w_{i j}-e_{i j}\right) \delta_{M_{i} M_{j}}$.
Equation 1-Louvain modularity algorithm, where $v$ is the total weight of the network (sum of all positive connections), $w_{i j}$ is the weighted connection between regions $i$ and $j, e_{i j}$ is the strength of a connection divided by the total weight of the network, and $\delta_{M i M j}$ is set to 1 when regions are in the same community and 0 otherwise.

Due to the stochastic nature of the Louvain algorithm, the community assignment for each region was assessed 100 times on the group average connectome and a consensus partition was identified using a fine-tuning algorithm. To define an appropriate value for the resolution parameter $(\gamma)$, we iterated the Louvain algorithm across a range of values (0.5-2.0 in steps of 0.1 ) for 100 iterations and then estimated the similarity of the resultant partitions using mutual information. A $\gamma$ parameter of 1.0 provided the most robust estimates of topology across these iterations and, thus, was used for the main experiment.

To estimate the topology at the regional level, we calculated the module degree $\mathrm{z}$ score $\left(W_{i}\right.$; a measure of intra-module connectivity; see Eq. 2) and participation coefficient ( $B_{i}$; a measure of inter-module connectivity; see Eq. 3) for each node. Positive $W_{i}$ values reflect nodes with more connections to other members of the same community (compared to the average number of connections) and vice versa. In contrast, the $B_{i}$ values ranges between 0 and 1 , with a value of 0 , indicating that all connections are within its community, whereas a value of 1 indicates uniformly distributed connections across modules (https://github.com/juliemaehall/topology):

$W_{i}=\frac{\kappa_{i}-\kappa_{s_{i}}^{\prime}}{\sigma_{\kappa_{s_{i}}}}$. 
Equation 2-Module degree $z$ score $W_{i}$, where $\kappa_{i}$ is the strength of the connections of region $i$ to other regions in its module $s_{i}, \kappa_{s_{i}}^{\prime}$ is the average of $\kappa$ over all the regions in $\mathrm{s}_{i}$, and $\sigma_{\kappa_{s_{i}}}$ is the standard deviation of $\kappa$ in $s_{i \text { : }}$

$B_{i}=1-\sum_{s=1}^{n_{M}}\left(\frac{\kappa_{i s}}{\kappa_{i}}\right)^{2}$

Equation 3-Participation coefficient $B_{i}$, where $\kappa_{i s}$ is the strength of the positive connections of region $i$ to regions in module $s$, and $\kappa_{\mathrm{i}}$ is the sum of strengths of all positive connections of region $i$. The participation coefficient of a region is, therefore, close to 1 if its connections are uniformly distributed among all the modules and 0 if all of its links are within its own module.

\section{Rich-club analysis}

We next identified the structural 'rich club' of the binarized group mean connectivity matrix of the entire sample. The 'rich club' of a network represents a set of high degree nodes (i.e. nodes with a large number of edges) that are more densely interconnected with other high degree nodes than would be expected by chance [33]. To estimate these regions, the degree of each node $i$ was determined by taking the sum of the number of connections node $i$ shared with $k$ other nodes in the network. Then, a sub-network (the ' $k$ core') was obtained by removing all nodes that had connections $\leq k$. For each value of $k$, the rich-club coefficient $\Phi_{k}$ was then computed as the ratio of the number of connections between the nodes in the $k$ core network and the sum of possible connections that would be present if the network was completely connected (see Eq. 4):

$\Phi_{k}=\frac{2 E_{>k}}{N_{>k}\left(N_{>k}-1\right)}$.

Since random networks also show an increasing function of $\Phi_{k}$, we next normalized the rich-club coefficient in reference to a null model. To this end, we created 5000 random networks with a preserved degree distribution, and then, the average rich-club coefficient $\Phi_{\text {rand }}$ over the set of random networks was calculated for each level of $k$. We then divided $\Phi_{k}$ by $\Phi_{\text {rand, }}$ and to define the normalized rich-club coefficient $\left(\Phi_{Z}\right)$. Values of $k$ with a $\Phi_{Z}>1$ can thus be said to contain a stronger rich-club architecture than expected under the conditions of the null model [33]. The identity of rich-club nodes for the highest value of $k$ with $\Phi_{\mathrm{Z}}>1$ can be found by identifying nodes that were present within the $k$ core network for that value of $k$. The 85th percentile of nodes was selected as part of the rich club.

\section{Statistical analyses}

Data analyses for demographic and clinical variables were performed using IBM SPSS Version 19. Independent $t$ tests and Mann-Whitney $U$ tests were performed, depending on the distribution of the data. Missing values for LEDD ( $n=1 ; 2 \%$ of the sample), Logical Memory $(n=1 ; 2 \%$ of the sample), $\mathrm{TMT}_{\mathrm{B}-\mathrm{A}}(n=2 ; 4 \%$ of the sample), and DBS $(n=1,2 \%$ of the sample) were replaced using the expectation minimization algorithm. The results are presented in Table 1. To assess statistical significance for the regional level metrics, a non-parametric permutation testing approach was used (5000 iterations) that effectively controls for multiple comparisons [34]. In addition, permutation testing with 5000 iterations was applied following a Spearman's correlation analysis between the significant $B_{i}$ and $W_{i}$ values and $\mathrm{TMT}_{\mathrm{B}-\mathrm{A}}$ and DSB scores.

To determine whether there was overlap between the rich club and regions with altered topology in our two clinical groups, we compared the spatial overlap between the three variables. To analyse whether this overlap was statistically significant, a permutation test was performed in which we randomly permuted the rich-club identity of each region (5000 iterations) and used the overlap between the randomized vector and the significantly different $W_{i}$ and $B_{i}$ scores to populate two separate null distributions. We then compared the empirical overlap between rich club and topological outcome measures with the null distributions and rejected the null hypothesis of no overlap if the true overlap was less than the 97.5th percentile of null distribution (i.e., the top $2.5 \%$ ).

The difference scores between the groups on the $B_{i}$ and $W_{i}$ values were compared across the three different thresholds, and moderate-to-very strong correlations were found, confirming the robustness of the findings (see Supplementary Materials).

\section{Results}

\section{Demographics}

Twenty-six patients were identified as freezers and twentyfour as non-freezers. There were no differences between the groups for age, disease duration, dopaminergic medication, disease severity as measured by the MDS-UPDRS part III and $\mathrm{H} \& \mathrm{Y}$ scale and the MMSE, Logical Memory, $\mathrm{TMT}_{\mathrm{B}-\mathrm{A}}$, and DSB (see Table 1).

\section{Graph metrics}

A small-world organisation of the structural brain networks was confirmed in both patient groups [33]. Table 2 
Table 2 Nodes with significantly different participation coefficients between groups

\begin{tabular}{|c|c|c|c|c|c|c|c|}
\hline Node & Region & $x$ & $y$ & $z$ & Freezers & Non-freezers & Rich club \\
\hline \multicolumn{8}{|l|}{ Non-freezers $>$ freezers } \\
\hline \multicolumn{8}{|l|}{ Subcortical } \\
\hline R caudate & Basal ganglia & 15 & 10 & 11 & $0.690 \pm 0.05$ & $0.731 \pm 0.05$ & $\checkmark$ \\
\hline $\mathrm{R}$ hippocampus & Hippocampus & 28 & -22 & -13 & $0.613 \pm 0.09$ & $0.668 \pm 0.08$ & $\checkmark$ \\
\hline $\mathrm{R}$ thalamus & Thalamus & 13 & -17 & 7 & $0.699 \pm 0.07$ & $0.752 \pm 0.05$ & $\checkmark$ \\
\hline \multicolumn{8}{|l|}{ Frontal } \\
\hline lh-lateralorbitofrontal_4 & Orbitofrontal cortex & -12 & 52 & -19 & $0.562 \pm 0.12$ & $0.628 \pm 0.10$ & - \\
\hline rh-lateralorbitofrontal_4 & Orbitofrontal cortex & 16 & 52 & -18 & $0.525 \pm 0.13$ & $0.612 \pm 0.10$ & $\checkmark$ \\
\hline lh-medialorbitofrontal_2 & Orbitofrontal cortex & -5 & 33 & -20 & $0.596 \pm 0.11$ & $0.676 \pm 0.07$ & - \\
\hline lh-superiorfrontal_5 & Prefrontal cortex & -11 & 36 & 47 & $0.594 \pm 0.10$ & $0.653 \pm 0.09$ & - \\
\hline lh-superiorfrontal_7 & Premotor cortex & -8 & 14 & 52 & $0.579 \pm 0.16$ & $0.660 \pm 0.08$ & - \\
\hline lh-superiorfrontal_8 & Premotor cortex & -8 & -2 & 63 & $0.524 \pm 0.13$ & $0.628 \pm 0.10$ & - \\
\hline lh-superiorfrontal_9 & Premotor cortex & -17 & -1 & 67 & $0.524 \pm 0.10$ & $0.608 \pm 0.09$ & - \\
\hline rh-superiorfrontal_3 & Prefrontal cortex & 11 & 40 & 40 & $0.580 \pm 0.16$ & $0.670 \pm 0.11$ & - \\
\hline rh-superiorfrontal_4 & Prefrontal cortex & 18 & 34 & 47 & $0.571 \pm 0.17$ & $0.674 \pm 0.09$ & - \\
\hline rh-superiorfrontal_5 & Premotor cortex & 12 & 18 & 52 & $0.583 \pm 0.14$ & $0.679 \pm 0.09$ & - \\
\hline rh-superiorfrontal_6 & Premotor cortex & 11 & 2 & 60 & $0.521 \pm 0.15$ & $0.611 \pm 0.09$ & - \\
\hline rh-paracentral_2 & Primary motor cortex & 10 & -16 & 59 & $0.199 \pm 0.18$ & $0.325 \pm 0.18$ & - \\
\hline \multicolumn{8}{|l|}{ Parietal } \\
\hline lh-inferiorparietal_4 & Somatosensory association cortex & -36 & -71 & 42 & $0.304 \pm 0.16$ & $0.423 \pm 0.18$ & - \\
\hline lh-postcentral_2 & Primary somatosensory cortex & -26 & -31 & 65 & $0.596 \pm 0.11$ & $0.676 \pm 0.10$ & - \\
\hline lh-postcentral_3 & Primary somatosensory cortex & -40 & -30 & 57 & $0.479 \pm 0.15$ & $0.565 \pm 0.16$ & - \\
\hline lh-superiorparietal_2 & Somatosensory association cortex & -33 & -43 & 55 & $0.239 \pm 0.16$ & $0.373 \pm 0.17$ & - \\
\hline lh-superiorparietal_4 & Somatosensory association cortex & -20 & -62 & 61 & $0.574 \pm 0.14$ & $0.681 \pm 0.08$ & $\checkmark$ \\
\hline lh-superiorparietal_7 & Visual association cortex & -13 & -87 & 34 & $0.548 \pm 0.15$ & $0.640 \pm 0.10$ & $\checkmark$ \\
\hline \multicolumn{8}{|l|}{ Cingulate } \\
\hline lh-posteriorcingulate_1 & Posterior cingulate gyrus & -4 & -11 & 35 & $0.409 \pm 0.16$ & $0.512 \pm 0.18$ & - \\
\hline rh-caudalanteriorcingulate_1 & Anterior cingulate gyrus & 9 & 36 & 5 & $0.497 \pm 0.17$ & $0.602 \pm 0.18$ & - \\
\hline \multicolumn{8}{|l|}{ Non-freezers $<$ freezers } \\
\hline rh-caudalmiddlefrontal_1 & Prefrontal cortex & 36 & 2 & 47 & $0.333 \pm 0.17$ & $0.235 \pm 0.16$ & - \\
\hline
\end{tabular}

lh Left hemisphere, $r h$ right hemisphere

and Fig. 2a demonstrate the 23 nodes that had significantly lower $B_{i}$ values in freezers compared to non-freezers (nodes coloured in green in Fig. 2a). Three of these more segregated nodes encompassed subcortical nuclei, including the right caudate, thalamus, and hippocampus. In the left hemisphere, we found six nodes in the frontal cortex, six nodes in the parietal cortex, and one node in the posterior cingulate gyrus. In the right hemisphere, six nodes in the frontal cortex and one node in the anterior cingulate gyrus showed significantly lower values in freezers compared to non-freezers. Conversely, the right caudal middle frontal gyrus showed a significantly higher $B_{i}$ value in freezers. Nine nodes in distributed cortical regions with lower $W_{i}$ scores (nodes coloured in green in Fig. 2b) and six cortical nodes with higher $\mathrm{z}$ scores (nodes coloured in orange in Fig. 2b) were found in PD freezers compared to non-freezers (see Table 3).
Of the regions that showed significantly different $B_{i}$ values, two nodes in the superior parietal cortex negatively correlated with the $\mathrm{TMT}_{\mathrm{B}-\mathrm{A}}$ scores ('lh-superiorparietal_2': $r=-0.32, p<0.05$; 'lh-superiorparietal_4': $r=-0.34$, $p<0.05$ ), whilst the right hippocampus and node 'rh-superiorfrontal_4' showed a positive correlation with the DSB ( $r=0.35, p<0.01$ and $r=0.27, p<0.05$, respectively). One node with a significantly different $W_{i}$ value showed a positive correlation ('lh-superiorfrontal_4': $r=0.30, p<0.05$ ) with the $\mathrm{TMT}_{\mathrm{B}-\mathrm{A}}$. Finally, node 'rh- superiorfrontal_8' showed a negative correlation with the DSB $(r=0.17, p<0.05)$, whilst node 'rh-rostralmiddlefrontal_4' showed a negative correlation with this test performance $(r=-0.27 p<0.05)$.

A rich-club network encompassing 35 nodes was identified. Of the 24 nodes that had a significantly different $B_{i}$ value between groups, six nodes were identified as part of the rich-club, which was deemed significant $(p<0.05$; see 


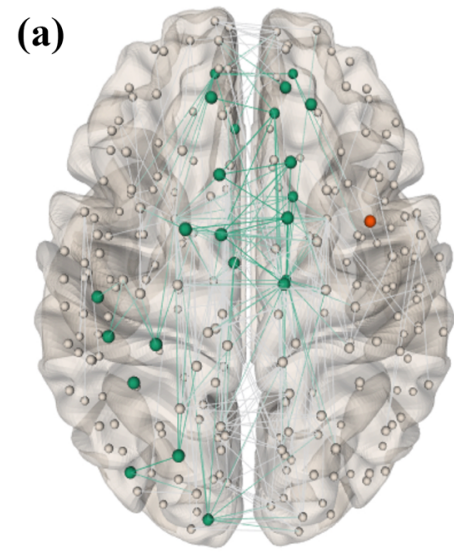

Nodes with significantly lower B-values in freezers
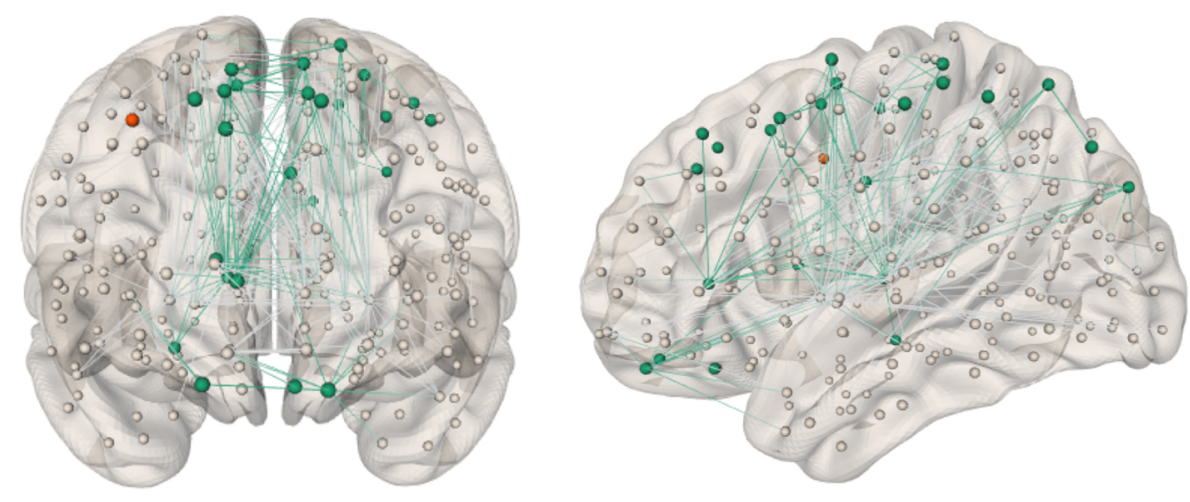

Nodes with significantly higher B-values in freezers

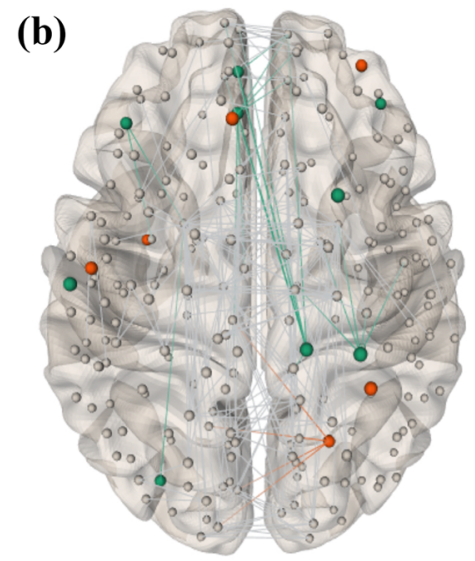

Nodes with significantly lower W-values in freezers
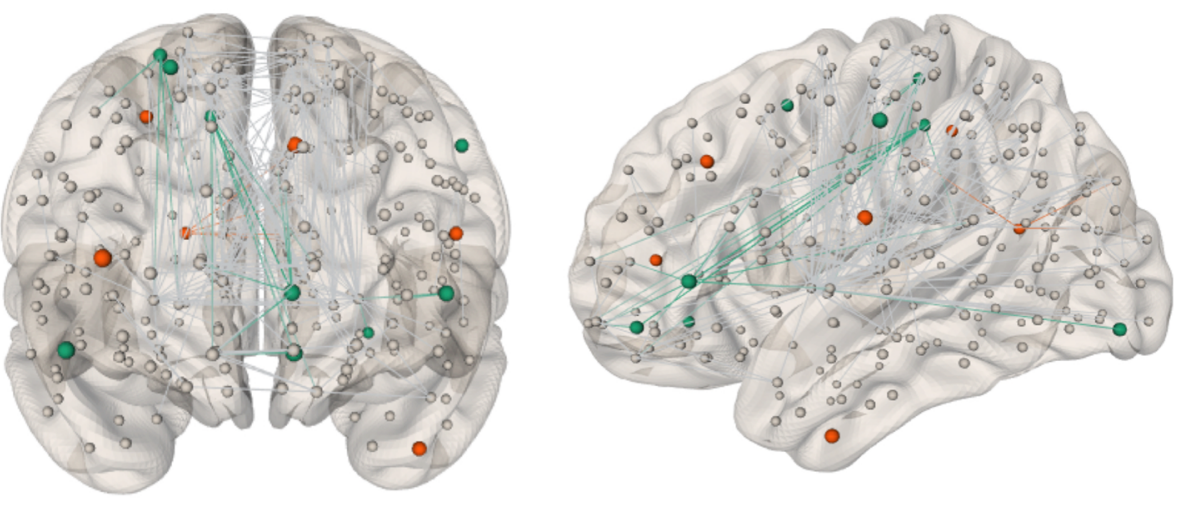

Nodes with significantly higher W-values in freezers

Fig. 2 a Nodes with a significantly different participation coefficient in freezers compared to non-freezers. b Nodes with a significantly different module degree $z$ score in freezers compared to non-freezers

Table 2, Fig. 3 and Supplementary materials). Of the 15 nodes with different $W_{i}$ values between groups, two nodes were considered within the rich-club network, but this overlap was not significant $(p>0.05)$.

\section{Discussion}

Here, we used graph theoretical methods to assess the structural network topology of PD patients with FOG. This approach allowed us to investigate both integrative as well as specialised properties of individual nodes within the brain's structural connectivity network in a data-driven manner, thus obviating any a priori assumptions about selected regions. In doing so, we demonstrated that freezers had a significantly lower participation coefficient in 23 of the 233 nodes compared to non-freezers. Specifically, these more segregated nodes included the right subcortical caudate, thalamus, and hippocampus, as well as nodes within bilateral frontal and parietal regions. Moreover, the six of these nodes were also part of the rich-club network that forms the backbone of the structural connectome by reducing path length (as opposed to multiple short-path connections) and hence permits faster and less noisy signal transmission [18, 33, 35].

Therefore, in keeping with other biological models [36, 37], we hypothesize that the symptoms of FOG may in part arise due to a lack of structural integration between key information processing hubs of the brain including motor, cognitive and limbic structures. A decreased ability to recruit alternative routes for parallel information processing [38] would lead to a lack of resilience when there are functional network challenges (such as cognitive dual-task performance) and also offer an explanation for the known characteristic set-shifting deficits in individuals with FOG [4]. The motor manifestation of FOG could thus arise due to a flow-on effect, whereby an inefficient network architecture would hamper the effective communication between major cortical and subcortical integration centres [7]. 
Table 3 Nodes with significantly different module degree z-scores between groups

\begin{tabular}{|c|c|c|c|c|c|c|}
\hline Node & Region & $x$ & $y$ & $z$ & Freezers & Non-freezers \\
\hline \multicolumn{7}{|l|}{ Non-freezers $>$ freezers } \\
\hline \multicolumn{7}{|l|}{ Frontal } \\
\hline lh-medialorbitofrontal_1 & Orbitofrontal cortex & -7 & 52 & -11 & $-0.482 \pm 0.70$ & $0.189 \pm 0.95$ \\
\hline lh-parstriangularis_1 & Prefrontal cortex & -43 & 33 & 2 & $0.371 \pm 0.74$ & $0.847 \pm 0.88$ \\
\hline rh-parsorbitalis_1 & Prefrontal cortex & 43 & 43 & -10 & $0.324 \pm 0.80$ & $0.904 \pm 0.66$ \\
\hline rh-superiorfrontal_8 & Prefrontal cortex & 22 & 10 & 58 & $-0.574 \pm 0.49$ & $-0.181 \pm 0.51$ \\
\hline \multicolumn{7}{|l|}{ Parietal } \\
\hline lh-postcentral_5 & Primary sensory cortex & -55 & -15 & 43 & $-0.686 \pm 0.48$ & $-0.367 \pm 0.52$ \\
\hline rh-postcentral_5 & Primary sensory cortex & 28 & -32 & 69 & $0.249 \pm 0.72$ & $0.826 \pm 0.91$ \\
\hline \multicolumn{7}{|l|}{ Occipital } \\
\hline lh-lateraloccipital_5 & Visual association cortex & -34 & -83 & -10 & $-0.222 \pm 0.80$ & $0.534 \pm 0.78$ \\
\hline \multicolumn{7}{|l|}{ Cingulate } \\
\hline lh-rostralanteriorcingulate_1 & Anterior cingulate & -7 & 37 & 2 & $0.566 \pm 1.00$ & $1.234 \pm 0.83$ \\
\hline rh-rostralanteriorcingulate_1 & Anterior cingulate & 14 & -33 & 52 & $0.713 \pm 0.93$ & $1.397 \pm 0.50$ \\
\hline \multicolumn{7}{|l|}{ Non-freezers $<$ freezers } \\
\hline \multicolumn{7}{|l|}{ Frontal } \\
\hline lh-superiorfrontal_4 & Prefrontal cortex & -8 & 32 & 37 & $-0.556 \pm 0.87$ & $-1.082 \pm 0.57$ \\
\hline rh-rostralmiddlefrontal_4 & Prefrontal cortex & 34 & 52 & 10 & $-0.837 \pm 0.54$ & $-1.187 \pm 0.55$ \\
\hline \multicolumn{7}{|l|}{ Parietal } \\
\hline lh-postcentral_7 & Retrosubicular area & -52 & -11 & 19 & $-0.354 \pm 0.75$ & $-0.724 \pm 0.52$ \\
\hline rh-superiorparietal_1 & Somatosensory association cortex & 33 & -43 & 54 & $0.550 \pm 0.85$ & $0.095 \pm 0.79$ \\
\hline rh-precuneus_1 & Visual association cortex & 23 & -65 & 21 & $0.282 \pm 0.76$ & $-0.183 \pm 0.71$ \\
\hline \multicolumn{7}{|l|}{ Temporal } \\
\hline lh-inferiortemporal_1 & Inferior temporal gyrus & -41 & -3 & -38 & $-0.100 \pm 0.61$ & $-0.518 \pm 0.68$ \\
\hline
\end{tabular}

lh left hemisphere, $r h$ right hemisphere

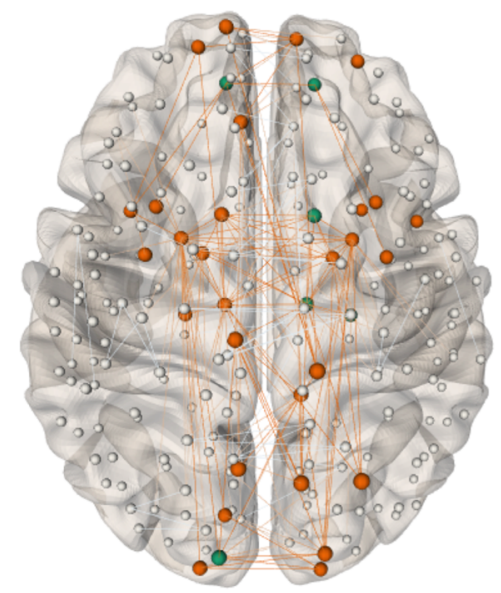

Rich club
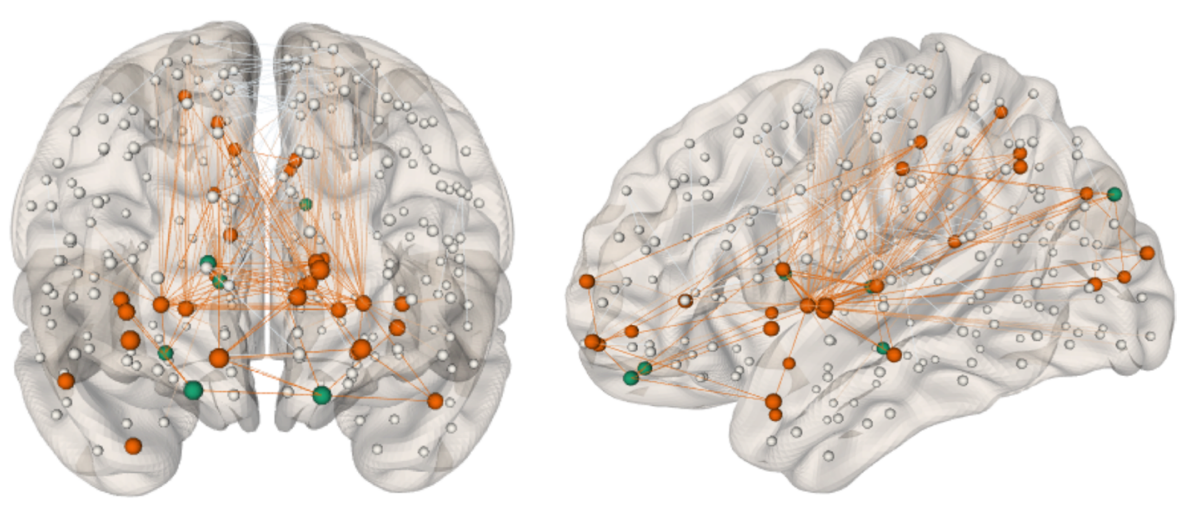

Fig. 3 Representation of the rich-club network

Nodes with high participating coefficients have previously been characterized as 'connector hubs' [39], as they tend to interconnect otherwise segregated communities. In the brain, nodes with high participation are thought to be crucial for incorporating complex information streams of different modalities. Affected participation scores, especially 
across these connector hubs, may cause disruptions extending across cognitive, motor, and limbic domains [18]. Nodes with high participation coefficients exhibit more activity when coordinated activity occurs across communities, such as during cognitive task performance [15]. These hubs provide an integrative network function to an even greater extent than rich-club nodes [40]; and importantly, ten regions affected in freezers were amongst the nodes with the highest participation scores. Hence, they likely play a crucial role in disrupted information processing in these patients.

The caudate nucleus and thalamus have previously been implicated in the freezing pathophysiology with studies showing reduced grey matter volume [41] and hypo-activity within subcortical structures [42]. Importantly, the thalamic nuclei receive input from and project to diffuse areas in the cortex, basal ganglia, and cerebellum which play a role in modulating activity in the rest of the brain. Rather than simply being a relay station, the thalamic nuclei are a major hub for controlling the information flow across cortical and subcortical regions [43]. The caudate nucleus also receives inputs from various prefrontal regions [44] and plays a major part in task-switching behaviour, which can be understood in terms of activating different modules for specialised function. Despite not reaching significance, the left thalamus and caudate also showed lower participation coefficients in freezers ( $p=0.07$ and $p=0.08$, respectively), and hence, there was no strong lateralization effect observed. Thus, the lower participation scores of these nodes may contribute to the pathophysiology of FOG through either ineffective integration of motor, sensory, and limbic activity during gait or inefficient shifting between functionally distinct but complementary neural pathways [7].

The previous findings of neuromodulatory systems have demonstrated the existence of an inverted U-shaped curve on executive functioning [36], and recent conceptual work has suggested that this relationship may also be present in network topology [15]. Consistent with the well-established association between FOG and impaired executive function [45-47], our findings showed less integrated nodes in the caudate, bilateral dorsolateral prefrontal (DLPFC), and anterior cingulate cortex. Moreover, participation coefficients of nodes within the DLPFC, superior parietal cortex, and hippocampus were associated with a performance on tasks investigating set-shifting and working memory. Thus, is it possible that changes in integration scores in areas associated with cognitive performance affect effective information transfer across the network, and might explain these phenotypic changes. However, the within the prefrontal cortex, one node showed a higher participation coefficienct, perhaps reflecting a rerouting of the common path in this region.

Lower participation coefficients were also found across frontoparietal structures, including the premotor, primary motor cortex, and somatosensory association cortices,
Automated movements are performed by activity in segregated modules $[15,17]$ and these movements are affected by PD pathology [48]. As such, PD patients rely more on effective communication in superior frontoparietal regions, since combining different sources of information between these regions is fundamental for successful completion of an accurate and context-appropriate movement [49]. When a region becomes too specialised or segregated, it loses its flexibility that is necessary to navigate through changing circumstances, which may underpin the difficulties which freezers experience navigating complex environments.

A number of fMRI studies using region-of-interest analyses comparing PD freezers to non-freezers have shown alterations in similar regions as reported in the current study. Specifically, a functional decoupling between the bilateral cognitive control network and the basal ganglia during freezing episodes has been reported during motor arrests provoked by a virtual reality paradigm [50]. Furthermore, using this paradigm, freezing episodes were associated with alterations in BOLD response within frontoparietal sensorimotor regions, basal ganglia, thalamus, and in the mesencephalic locomotor region [42]. Altered resting-state functional connectivity within the cognitive control network, visual networks, and locomotor regions has further been shown to be associated with FOG severity [13, 51]. However, future research avenues should explore alterations in functional network topology associated with freezing of gait.

The current study also compared within-module connectivity across groups. Segregation into isolated modules allows for specialised processing of an action and increases efficiency, flexibility, and robustness to perturbation [35]. Abnormal within-module scores can affect these characteristics by increasing effective metabolic costs [35]. Compared to non-freezers, freezers showed lower module degree $z$ scores in nodes within the prefrontal cortex (pars orbitalis, pars triangularis, and orbitofrontal and rostral anterior cingulate), primary sensory, primary motor, and visual association cortex. Perhaps surprisingly, higher module degree $\mathrm{z}$ scores were observed in patients with FOG in prefrontal cortices (DLPFC and the frontal eye fields), and various sensory information processing areas such as the somatosensory association, visual association, and inferior temporal regions. Interestingly, higher module degree z scores are considered favourable for behaviours that require specific, unimodal function, such as certain sensory processing systems [52]. As such, freezers may need to rely more heavily on exogenous stimuli to gather information to guide their actions, perhaps, due to a loss of automaticity [53]. Indeed, the previous studies have shown a proprioceptive deficit in PD patients with [54] and without freezing [49] along with a greater dependence on visual information [55]. Therefore, it could be speculated that a reweighting of sensory systems occurs in PD patients with freezing. Specifically, there may 
be an increased emphasis placed on discrete modality specific pathways that process afferent information; however, if the system is unable to effectively integrate this information, the dynamic evolution of the gait pattern may suffer.

\section{Limitations and future directions}

The current study has several limitations. First, we grouped the participants based on a subjective question of the FOGQ. Currently, there is no gold standard to define FOG, and whilst objective measures are preferable, FOG can be difficult to elicit in a clinical or experimental environment and might remain unnoticed [56]. Another possible weakness of this study's methodology is that the Louvain algorithm does not allow for overlapping modules, as a node is only assigned to one community. Furthermore, although DTI is an established method for measuring white matter integrity and has led to great insights in PD [57, 58], it suffers from inherent limitations. Specifically, diffusion imaging measures the motion of water molecules along a tract and thus only allows for indirect validation of streamlines. The deterministic diffusion tensor fitting approach used in this study requires sufficient directional information at each voxel and is incapable of resolving crossing or 'kissing' fibres. However, the deterministic diffusion tensor fitting approach used in this study requires sufficient directional information at each voxel and is incapable of resolving crossing or 'kissing' fibres. At an ambiguous directional point in a voxel, fractional anisotropy values might drop leading to a premature termination of the reconstruction of a tract. This could be especially problematic in structures with complex fibre architecture such as subcortical nuclei. Future research should use more sophisticated methods, such as constrained spherical deconvolution, that are better equipped to deal with complex fibre configurations. Finally, this study used single shell DTI data, without EPI distortion corrections, which could have impacted the registration accuracy between the DTI and T1 images, creating suboptimal connectomes. Hence, these results should be interpreted with caution and replication studies using multi-shell sequences are necessary to fully understand the changes in network topology associated with FOG.

\section{Conclusion}

This is the first study to use graph theory to demonstrate subtle alterations in network topology in PD patients with FOG compared to those without this symptom. Specifically, we showed that freezers have lower participation scores in subcortical regions as well as higher order and integrative cortical cortices. We argue that this increase in segregation hinders efficient communication between major cortical and subcortical centres, resulting in ineffective integration of motor, sensory, and limbic activity, as well as inefficient shifting between specialised modules that could disrupt gait in PD. Decreased intra-module connectivity might reflect a less cost-effective processing of information, whereas an increase in these scores in the sensory cortex of freezers suggests a possible compensatory strategy.

Acknowledgements We thank the patients and their families who contribute to our research at the Parkinson's Disease Research Clinic. This research was supported by Sydney Informatics Hub, funded by the University of Sydney. JMH is supported by a Western Sydney University Postgraduate Research Award; JMS is supported by a National Health and Medical Research Council CJ Martin Fellowship (1072403); KAEM is supported by a Parkinson Canada Fellowship; MG is supported by a University of Sydney International Scholarship; SJGL is supported by National Health and Medical Research CouncilAustralian Research Council Dementia Fellowship (\#1110414) and this work was supported by funding to Forefront, a collaborative research group dedicated to the study of non-Alzheimer disease degenerative dementias, from the National Health and Medical Research Council of Australia program grant (\#1037746 and \#1095127). KMB, JYYS, $\mathrm{CCW}$, and AAM have no funding source to disclose.

\section{Compliance with ethical standards}

Conflict of interest The authors declare that they have no conflict of interest.

Ethical standards All persons gave their informed consent prior to their inclusion in registries. The registries were approved by the Human Research Ethics Committee of the University of Sydney. Patients were included after informed written consent had been obtained, as set forth by the Declaration of Helsinki (WMA, 1964-2014).

\section{References}

1. Nutt JG, Bloem BR, Giladi N, Hallett M, Horak FB, Nieuwboer A (2011) Freezing of gait: moving forward on a mysterious clinical phenomenon. Lancet Neurol 10(8):734-744. https://doi. org/10.1016/S1474-4422(11)70143-0

2. Walton CC, Shine JM, Hall JM, O'Callaghan C, Mowszowski L, Gilat M, Szeto JY, Naismith SL, Lewis SJ (2015) The major impact of freezing of gait on quality of life in Parkinson's disease. J Neurol 262(1):108-115. https://doi.org/10.1007/s0041 5-014-7524-3

3. Plotnik M, Giladi N, Hausdorff JM (2012) Is freezing of gait in Parkinson's disease a result of multiple gait impairments? Implications for treatment. Parkinson's Dis 2012:8. https://doi. org/10.1155/2012/459321

4. Naismith SL, Shine JM, Lewis SJG (2010) The specific contributions of set-shifting to freezing of gait in Parkinson's disease. Mov Disord 25(8):1000-1004. https://doi.org/10.1002/mds.23005

5. Ehgoetz Martens KA, Ellard CG, Almeida QJ (2014) A closer look at mechanisms underlying perceptual differences in Parkinson's freezers and non-freezers. Neuroscience 274:162-169. https ://doi.org/10.1016/j.neuroscience.2014.05.022

6. Ehgoetz Martens KA, Ellard CG, Almeida QJ (2014) Does anxiety cause freezing of gait in Parkinson's disease? PLoS One 9(9):e106561. https://doi.org/10.1371/journal.pone.0106561 
7. Lewis SJ, Shine JM (2016) The next step: a common neural mechanism for freezing of gait. Neuroscientist 22(1):72-82. https://doi. org/10.1177/1073858414559101

8. Schweder PM, Hansen PC, Green AL, Quaghebeur G, Stein J, Aziz TZ (2010) Connectivity of the pedunculopontine nucleus in parkinsonian freezing of gait. NeuroReport 21(14):914-916. https ://doi.org/10.1097/WNR.0b013e32833ce5f1

9. Fling BW, Cohen RG, Mancini M, Nutt JG, Fair DA, Horak FB (2013) Asymmetric pedunculopontine network connectivity in parkinsonian patients with freezing of gait. Brain 136(Pt 8):24052418. https://doi.org/10.1093/brain/awt172

10. Vercruysse S, Leunissen I, Vervoort G, Vandenberghe W, Swinnen S, Nieuwboer A (2015) Microstructural changes in white matter associated with freezing of gait in Parkinson's disease. Mov Disord 30(4):567-576. https://doi.org/10.1002/mds.26130

11. Youn J, Lee JM, Kwon H, Kim JS, Son TO, Cho JW (2015) Alterations of mean diffusivity of pedunculopontine nucleus pathway in Parkinson's disease patients with freezing of gait. Parkinsonism Rel Disord 21(1):12-17. https://doi.org/10.1016/j.parkreldis .2014.10.003

12. Pietracupa S, Suppa A, Upadhyay N, Giannì C, Grillea G, Leodori G, Modugno N, Di Biasio F, Zampogna A, Colonnese C, Berardelli A, Pantano P (2018) Freezing of gait in Parkinson's disease: gray and white matter abnormalities. J Neurol 265(1):52-62. https://doi.org/10.1007/s00415-017-8654-1

13. Fling BW, Cohen RG, Mancini M, Carpenter SD, Fair DA, Nutt JG, Horak FB (2014) functional reorganization of the locomotor network in Parkinson patients with freezing of gait. PLoS One 9(6):e100291. https://doi.org/10.1371/journal.pone.0100291 (ARTN)

14. Canu E, Agosta F, Sarasso E, Volonte MA, Basaia S, Stojkovic T, Stefanova E, Comi G, Falini A, Kostic VS, Gatti R, Filippi M (2015) Brain structural and functional connectivity in Parkinson's disease with freezing of gait. Hum Brain Mapp 36(12):50645078. https://doi.org/10.1002/hbm.22994

15. Shine JM, Poldrack RA (2017) Principles of dynamic network reconfiguration across diverse brain states. NeuroImage. https:// doi.org/10.1016/j.neuroimage.2017.08.010

16. Sporns O, Betzel RF (2016) Modular brain networks. Annu Rev Psychol 67:613-640. https://doi.org/10.1146/annurev-psych $-122414-033634$

17. Bassett DS, Yang M, Wymbs NF, Grafton ST (2015) Learning-induced autonomy of sensorimotor systems. Nat Neurosci 18(5):744-751. https://doi.org/10.1038/nn.3993

18. Bertolero MA, Yeo BTT, D'Esposito M (2015) The modular and integrative functional architecture of the human brain. Proc Natl Acad Sci 112(49):E6798-E6807. https://doi.org/10.1073/ pnas. 1510619112

19. Sporns O, Chialvo DR, Kaiser M, Hilgetag CC (2004) Organization, development and function of complex brain networks. Trends Cogn Sci 8(9):418-425. https://doi.org/10.1016/j.tics.2004.07.008

20. Goetz CG, Tilley BC, Shaftman SR, Stebbins GT, Fahn S, Martinez-Martin P, Poewe W, Sampaio C, Stern MB, Dodel R, Dubois B, Holloway R, Jankovic J, Kulisevsky J, Lang AE, Lees A, Leurgans S, LeWitt PA, Nyenhuis D, Olanow CW, Rascol O, Schrag A, Teresi JA, van Hilten JJ, LaPelle N, Movement Disorder Society URTF (2008) Movement Disorder Society-sponsored revision of the Unified Parkinson's Disease Rating Scale (MDS-UPDRS): scale presentation and clinimetric testing results. Mov Disord 23(15):2129-2170. https://doi.org/10.1002/mds.22340

21. Hoehn MM, Yahr MD (1967) Parkinsonism: onset, progression and mortality. Neurology 17(5):427-442

22. Folstein MF, Folstein SE, McHugh PR (1975) "Mini-mental state". A practical method for grading the cognitive state of patients for the clinician. J Psychiatr Res 12(3):189-198
23. Wechsler DS (1997) Wechsler memory scale. The Psychological Corporation, San Antonio

24. Army Battery IT (1944) Manual of directions and scoring. War Department, Adjutant General's Office, Washington, DC

25. Giladi N, Shabtai H, Simon ES, Biran S, Tal J, Korczyn AD (2000) Construction of freezing of gait questionnaire for patients with Parkinsonism. Parkinsonism Rel Disord 6(3):165-170

26. Shine JM, Moore ST, Bolitho SJ, Morris TR, Dilda V, Naismith SL, Lewis SJG (2012) Assessing the utility of freezing of Gait Questionnaires in Parkinson's disease. Parkinsonism Rel Disord 18(1):25-29. https://doi.org/10.1016/j.parkreldis.2011.08.002

27. Cammoun L, Gigandet X, Meskaldji D, Thiran JP, Sporns O, Do KQ, Maeder P, Meuli R, Hagmann P (2012) Mapping the human connectome at multiple scales with diffusion spectrum MRI. J Neurosci Methods 203(2):386-397. https://doi.org/10.1016/j. jneumeth.2011.09.031

28. Daducci A, Gerhard S, Griffa A, Lemkaddem A, Cammoun L, Gigandet X, Meuli R, Hagmann P, Thiran J-P (2012) The connectome mapper: an open-source processing pipeline to map connectomes with MRI. PLoS One 7(12):e48121. https://doi. org/10.1371/journal.pone.0048121

29. Fischl B, Salat DH, Busa E, Albert M, Dieterich M, Haselgrove C, van der Kouwe A, Killiany R, Kennedy D, Klaveness S, Montillo A, Makris N, Rosen B, Dale AM (2002) Whole brain segmentation: automated labeling of neuroanatomical structures in the human brain. Neuron 33(3):341-355

30. Zalesky A, Fornito A, Cocchi L, Gollo LL, van den Heuvel MP, Breakspear M (2016) Connectome sensitivity or specificity: which is more important? NeuroImage 142:407-420. https://doi. org/10.1016/j.neuroimage.2016.06.035

31. de Reus MA, van den Heuvel MP (2013) Estimating false positives and negatives in brain networks. NeuroImage 70:402-409. https://doi.org/10.1016/j.neuroimage.2012.12.066

32. Rubinov M, Sporns O (2010) Complex network measures of brain connectivity: uses and interpretations. NeuroImage 52(3):10591069. https://doi.org/10.1016/j.neuroimage.2009.10.003

33. van den Heuvel MP, Sporns O (2011) Rich-club organization of the human connectome. J Neurosci 31(44):15775-15786. https:// doi.org/10.1523/jneurosci.3539-11.2011

34. Nichols TE, Holmes AP (2002) Nonparametric permutation tests for functional neuroimaging: a primer with examples. Hum Brain Mapp 15(1):1-25. https://doi.org/10.1002/hbm.1058

35. Bullmore E, Sporns O (2012) The economy of brain network organization. Nat Rev Neurosci 13(5):336-349. https://doi. org/10.1038/nrn3214

36. Robbins TW, Arnsten AFT (2009) The neuropsychopharmacology of fronto-executive function: monoaminergic modulation. Ann Rev Neurosci 32:267-287. https://doi.org/10.1146/annur ev.neuro.051508.135535

37. Crossley NA, Mechelli A, Scott J, Carletti F, Fox PT, McGuire P, Bullmore ET (2014) The hubs of the human connectome are generally implicated in the anatomy of brain disorders. Brain 137(Pt 8):2382-2395. https://doi.org/10.1093/brain/awu132

38. Achard S, Salvador R, Whitcher B, Suckling J, Bullmore E (2006) A resilient, low-frequency, small-world human brain functional network with highly connected association cortical hubs. J Neurosci 26(1):63-72. https://doi.org/10.1523/JNEUR OSCI.3874-05.2006

39. Guimera R, Amaral LA (2005) Cartography of complex networks: modules and universal roles. J Stat Mech 2005(P02001):nihpa35573. https://doi.org/10.1088/17425468/2005/02/p02001

40. Bertolero MA, Yeo BTT, D'Esposito M (2017) The diverse club. Nat Commun 8(1):1277. https://doi.org/10.1038/s41467-01701189-w 
41. Herman T, Rosenberg-Katz K, Jacob Y, Giladi N, Hausdorff JM (2014) Gray matter atrophy and freezing of gait in Parkinson's disease: is the evidence black-on-white? Mov Disord 29(1):134-139. https://doi.org/10.1002/mds.25697

42. Shine JM, Matar E, Ward PB, Bolitho SJ, Gilat M, Pearson M, Naismith SL, Lewis SJG (2013) Exploring the cortical and subcortical fMRI changes associated with freezing in Parkinson's disease. Brain 136(4):1204-1215

43. Sherman SM (2016) Thalamus plays a central role in ongoing cortical functioning. Nat Neurosci 16(4):533-541. https://doi. org/10.1038/nn.4269

44. Jarbo K, Verstynen TD (2015) Converging structural and functional connectivity of orbitofrontal, dorsolateral prefrontal, and posterior parietal cortex in the human striatum. J Neurosci 35(9):3865-3878. https://doi.org/10.1523/jneurosci.2636-14.2015

45. Hall JM, Shine JM, O'Callaghan C, Walton CC, Gilat M, Naismith SL, Lewis SJG (2015) Freezing of gait and its associations in the early and advanced clinical motor stages of Parkinson's disease: a cross-sectional study. J Parkinson Dis 5(4):881-891. https://doi.org/10.3233/Jpd-150581

46. Vandenbossche J, Deroost N, Soetens E, Zeischka P, Spildooren J, Vercruysse S, Nieuwboer A, Kerckhofs E (2012) Conflict and freezing of gait in Parkinson's disease: support for a response control deficit. Neuroscience 206:144-154. https://doi.org/10.1016/j. neuroscience.2011.12.048

47. Walton CC, O'Callaghan C, Hall JM, Gilat M, Mowszowski L, Naismith SL, Burrell JR, Shine JM, Lewis SJG (2015) Antisaccade errors reveal cognitive control deficits in Parkinson's disease with freezing of gait. J Neurol 262(12):2745-2754. https://doi. org/10.1007/s00415-015-7910-5

48. Redgrave P, Rodriguez M, Smith Y, Rodriguez-Oroz MC, Lehericy S, Bergman H, Agid Y, DeLong MR, Obeso JA (2010) Goal-directed and habitual control in the basal ganglia: implications for Parkinson's disease. Nat Rev Neurosci 11(11):760-772

49. Wu T, Hallett M (2005) A functional MRI study of automatic movements in patients with Parkinson's disease. Brain $128(\mathrm{Pt}$ 10):2250-2259. https://doi.org/10.1093/brain/awh569
50. Shine JM, Matar E, Ward PB, Frank MJ, Moustafa AA, Pearson M, Naismith SL, Lewis SJ (2013) Freezing of gait in Parkinson's disease is associated with functional decoupling between the cognitive control network and the basal ganglia. Brain 136(Pt 12):3671-3681. https://doi.org/10.1093/brain/awt272

51. Tessitore A, Amboni M, Esposito F, Russo A, Picillo M, Marcuccio L, Pellecchia MT, Vitale C, Cirillo M, Tedeschi G, Barone P (2012) Resting-state brain connectivity in patients with Parkinson's disease and freezing of gait. Parkinsonism and Rel Disord 18(6):781-787. https://doi.org/10.1016/j.parkreldis.2012.03.018

52. Park HJ, Friston K (2013) Structural and functional brain networks: from connections to cognition. Science 342(6158):1238411. https://doi.org/10.1126/science.1238411

53. Vandenbossche J, Deroost N, Soetens E, Coomans D, Spildooren J, Vercruysse S, Nieuwboer A, Kerckhofs E (2012) Freezing of gait in Parkinson's disease: disturbances in automaticity and control. Front Hum Neurosci 6:356. https://doi.org/10.3389/fnhum .2012 .00356

54. Ehgoetz Martens KA, Pieruccini-Faria F, Almeida QJ (2013) Could sensory mechanisms be a core factor that underlies freezing of gait in Parkinson's disease? PLoS One 8(5):e62602. https ://doi.org/10.1371/journal.pone.0062602

55. Davidsdottir S, Cronin-Golomb A, Lee A (2005) Visual and spatial symptoms in Parkinson's disease. Vision Res 45(10):12851296. https://doi.org/10.1016/j.visres.2004.11.006

56. Nieuwboer A, De Weerdt W, Dom R, Lesaffre E (1998) A frequency and correlation analysis of motor deficits in Parkinson patients. Disabil Rehabil 20(4):142-150

57. de Oliveira RV, Pereira JS (2017) The role of diffusion magnetic resonance imaging in Parkinson's disease and in the differential diagnosis with atypical parkinsonism. Radiol Bras 50(4):250-257. https://doi.org/10.1590/0100-3984.2016-0073

58. Hall JM, Ehgoetz Martens KA, Walton CC, O'Callaghan C, Keller PE, Lewis SJ, Moustafa AA (2016) Diffusion alterations associated with Parkinson's disease symptomatology: a review of the literature. Parkinsonism Rel Disord 33:12-26. https://doi. org/10.1016/j.parkreldis.2016.09.026 